TRANSACTIONS OF THE

AMERICAN MATHEMATICAL SOCIETY

Volume 275, Number 2, February 1983

\title{
A SPHERE THEOREM FOR MANIFOLDS OF POSITIVE RICCI CURVATURE
}

\author{
BY
}

KATSUHIRO SHIOHAMA

Dedicated to Professor S. Sasaki on his 70 th birthday

\begin{abstract}
Instead of injectivity radius, the contractibility radius is estimated for a class of complete manifolds such that $\operatorname{Ric}_{M} \geqslant 1, K_{M} \geqslant-\kappa^{2}$ and the volume of $M \geqslant$ the volume of the $(\pi-\varepsilon)$-ball on the unit $m$-sphere, $m=\operatorname{dim} M$. Then for a suitable choice of $\varepsilon=\varepsilon(m, \kappa)$ every $M$ belonging to this class is homeomorphic to $S^{m}$.
\end{abstract}

1. Introduction. An important problem in Riemannian geometry is to investigate relations between curvature and topology of Riemannian manifolds. A classical and beautiful theorem due to Myers [14] states that if the Ricci curvature $\operatorname{Ric}_{M}$ of a complete Riemannian manifold $M$ satisfies $\operatorname{Ric}_{M} \geqslant 1$, then the diameter $d(M)$ of $M$ is not greater than $\pi$ and hence $M$ is compact and the fundamental group $\pi_{1}(M)$ of $M$ is finite. After the pioneering work of Rauch [15], the so-called rigidity theorem was proved by Berger [1] for even dimensional complete simply connected $M$ with the sectional curvature $\frac{1}{4} \leqslant K_{M} \leqslant 1$, and the so-called sphere theorem was proved by Klingenberg [13] which states that a complete simply connected $M$ with $\frac{1}{4}<K_{M} \leqslant 1$ is homeomorphic to a sphere. Recently the sphere theorem has been generalized by Grove and the author in [12].

Hewever very little has been known for the topology of complete manifolds of prsitive Ricci curvature. A splitting theorem due to Cheeger and Gromoll [6] states that if $M$ is complete noncompact with nonnegative Ricci curvature and if $M$ admits a straight line, then $M$ is isometric to the Riemannian product $M^{\prime} \times R$. Recently Schoen and Yau has proved in [17] that a 3 dimensional complete noncompact $M$ with positive Ricci curvature is diffeomorphic to $R^{3}$. Making use of the first eigenvalue for the Laplacian operator, Cheng [7] has proved that if $M$ is complete and if $\mathrm{Ric}_{M} \geqslant 1$ and if $d(M)=\pi$, then $M$ is isometric to the standard unit sphere.

Since Cheng's method is not useful for perturbation of metrics, it was expected to obtain an elementary and geometric approach for the proof of the above theorem. An elementary proof will make it possible to relax assumptions to replace isometry by homeomorphism or possibly by diffeomorphism.

The purpose of the present paper is to give first of all an elementary proof of the maximal diameter theorem. This is done by using the monotone property of the volume rate between concentric metric balls on $M$ and on the complete simply

Received by the editors November 17, 1981 and, in revised form, February 12, 1982.

1980 Mathematics Subject Classification. Primary 53C20; Secondary 58E10.

c 1983 American Mathematical Society 0002-9947/82/0000-0967/\$05.50 
connected space form of constant sectional curvature which is equal to the infimum of $\operatorname{Ric}_{M}$. This property was first obtained by Bishop (see [2,11.10]) for balls inside cut locus of their center and has recently been proved by Gromov (without giving details in [11]) beyond the cut locus of the center. Gromov used this property to obtain a uniform upper bound for the sum of Betti numbers for certain classes of complete Riemannian manifolds [11]. In the next place, an estimate of the radii of contractible metric balls on $M$ is obtained under certain conditions for Ricci and sectional curvatures on $M$. We then prove the

MaIn Theorem. Let $m$ be a positive integer and let $\kappa>0$ be a constant. Then there exists an $\varepsilon(m, \kappa)>0$ such that if $M$ is an m-dimensional complete manifold whose Ricci and sectional curvatures satisfy $\operatorname{Ric}_{M} \geqslant 1, K_{M} \geqslant-\kappa^{2}$, and if the volume $v(M)$ of $M$ satisfies $v(M) \geqslant \eta(m, \pi-\varepsilon(m, \kappa))$, then $M$ is homeomorphic to $S^{m}$, where $\eta(m, r)$ is the volume of the r-ball on $S^{m}(1)$.

The author does not know whether the assumption for the sectional curvature is essentially needed. It is a rather technical one from which the radius of contractible metric balls (instead of injectivity radius of exponential map) is estimated.

Note also that $v(M) \geqslant \eta(m, \pi-\varepsilon)$ and $\operatorname{Ric}_{M} \geqslant 1$ imply that the diameter $d(M)$ of $M$ takes value in $\pi-\varepsilon \leqslant d(M) \leqslant \pi$. However it is not certain whether the assumptions $\operatorname{Ric}_{M} \geqslant 1$ and $d(M) \geqslant \pi-\varepsilon$ will give a lower bound for the volume of $M$.

The proof of our main theorem is based on the generalized Schoenflies theorem due to Brown [3]. Namely, if $M$ is covered by two open disks, then $M$ is homeomorphic to $S^{m}$ (for details see Theorem 1.8 .4 on p. 49, [16]). The proof is achieved by covering $M$ by two contractible metric balls. Thus we prove

TheOREM 3.4. Let $m$ be a positive integer and let $\kappa>0$ and $\varepsilon \in(0, \pi / 3)$ be given constants. Then there exists for a fixed number $\delta \in[\varepsilon, \pi / 3)$ a constant $c_{\delta}(m, \kappa, \varepsilon)>0$ such that if $M$ is an m-dimensional complete manifold whose Ricci and sectional curvatures and volume satisfy $\operatorname{Ric}_{M} \geqslant 1, K_{M} \geqslant-\kappa^{2}$, and $v(M) \geqslant \eta(m, \pi-\varepsilon)$, then every point $x$ on $M$ has a contractible metric ball $B_{r}(x)$ around it with $r \geqslant c_{\delta}(m, \kappa, \varepsilon)$.

The rest of the paper is organized as follows. In $\$ 2$ an elementary proof of the Cheng maximal diameter theorem is given by using the basic lemma by Gromov [11]. With the aid of the Toponogov theorem, Theorem 3.4 is proved in $\S 3$. Finally the proof of our main theorem is stated in $\$ 4$.

An interesting problem is if a finiteness of homotopy types of the class of complete manifolds whose curvatures and volume fulfill $\mathrm{Ric}_{M} \geqslant 1, K_{M} \geqslant-\kappa^{2}$ and $v(M) \geqslant \eta(m, \pi-\varepsilon)$ can be proved, where $m, \kappa>0$ and $\varepsilon \in(0, \pi / 2)$ are constants. If $M$ satisfies the above conditions, then $M$ is covered by at most $N$ contractible metric balls, where $N$ depends only on $m, \kappa$ and $\varepsilon$. The problem reduces to the following: Let $M_{1}$ and $M_{2}$ be $m$-dimensional manifolds each $M_{i}$ of which is covered by $N^{\prime}(\leqslant N)$ topological disks $B_{1}^{i}, \ldots, B_{N^{\prime}}^{i}$ which are all metric balls of the same radius such that the number of components $B_{j_{1}}^{1} \cap \cdots \cap B_{j_{k}}^{1}$ is equal to that of $B_{j_{1}}^{2} \cap \cdots \cap B_{j_{k}}^{2}$ for all $j_{1}, \ldots, j_{k}=1, \ldots, N^{\prime}$. Then are they homotopy equivalent to 
each other? In the general case where they are not metric balls but topological disks, the answer is negative, as stated at the end of $\S 4$.

The author would like to express his thanks to T. Kaneto who let him know such a simple example.

2. An elementary proof of the Cheng theorem. Let $M$ be a complete Riemannian manifold of dimension $m$ and let $\operatorname{Ric}_{M} \geqslant-\kappa^{2}$, where $\kappa$ is a real or a pure imaginary number. Let $M\left(-\kappa^{2}\right)$ be the complete simply connected $m$-dimensional space form of constant sectional curvature $-\kappa^{2}$. For a point $x \in M$ and for an $r>0$ let $B_{r}(x)$ be the metric $r$-ball centered at $x$. A metric $r$-ball in $M\left(-\kappa^{2}\right)$ is denoted by $\tilde{B}_{r}$. With these notations the basic lemma due to Gromov is stated as

Lemma (Gromov [11]). For any fixed $x \in M$, let $f:[0, \infty) \rightarrow R$ be defined as

$$
f(r):=v\left(B_{r}(x)\right) / v\left(\tilde{B}_{r}\right),
$$

where $v(A)$ is by definition the volume of a set $A$. Then $f$ is monotone nonincreasing.

Making use of this lemma, we shall establish an elementary proof of the maximal diameter theorem due to Cheng [7], which is a generalization of the Toponogov theorem for sectional curvature [18].

TheOREM (S. Y. CHENG [7]). Let $M$ be a complete Riemannian m-manifold whose Ricci curvature is bounded below by 1 . If the diameter of $M$ is equal to $\pi$, then $M$ is isometric to $S^{m}(1)$.

Proof. Take a pair of points $p, q$ at maximal distance, $d(p, q)=d(M)=\pi$, where $d$ is the distance function induced from the Riemannian metric. Define the volume rate function $f_{p}, f_{q}:[0, \pi] \rightarrow R$ by $f_{p}(r)=v\left(B_{r}(p)\right) / v\left(\tilde{B}_{r}\right)$ and $f_{q}(r)=$ $v\left(B_{r}(q)\right) / v\left(\tilde{B}_{r}\right)$. It follows from assumption that $\bar{B}_{\pi / 2}(p) \cup \bar{B}_{\pi / 2}(q) \subset M$ and $B_{\pi / 2}(p) \cap B_{\pi / 2}(q)=\varnothing$, where $\bar{A}$ is by definition the closure of a set $A \subset M$. Thus $v(M) \geqslant v\left(\bar{B}_{\pi / 2}(p)\right)+v\left(\bar{B}_{\pi / 2}(q)\right)$.

On the other hand since the basic lemma implies that $f_{p}$ and $f_{q}$ are monotone nonincreasing, $f_{p}(\pi / 2)=v\left(B_{\pi / 2}(p)\right) /\left(c_{m} / 2\right) \geqslant f_{p}(\pi)=v(M) / c_{m}$. Hence $v\left(B_{\pi / 2}(p)\right) \geqslant v(M) / 2$, and similarly $v\left(B_{\pi / 2}(q)\right) \geqslant v(M) / 2$. This fact implies that $f_{p}(\pi / 2)=f_{p}(\pi)=f_{q}(\pi / 2)=f_{q}(\pi)$. It follows from the monotone property of $f_{p}$ that $f_{p}(r)=f_{p}(\pi)$ for all $r \in[\pi / 2, \pi]$. From $v(M) \geqslant v\left(B_{r}(p)\right)+v\left(B_{\pi-r}(q)\right)$ for $r \in$ $[\pi / 2, \pi]$ it follows that $f_{q}(\pi-r)=v\left(B_{\pi-r}(q)\right) / v\left(\tilde{B}_{\pi-r}\right) \geqslant f_{q}(\pi / 2)=f_{p}(\pi)$. Therefore $v(M) \geqslant v\left(B_{r}(p)\right)+v\left(B_{\pi-r}(q)\right) \geqslant f_{p}(\pi) \cdot c_{m}=v(M)$, and $f_{q}(\pi-r)=f_{q}(\pi / 2)$ for any $r \in[\pi / 2, \pi]$. Since $f_{p}(0)=f_{q}(0)=1$, this fact means that $f_{p}=f_{q}=1$, and hence $C_{p}=C_{q}=$ the sphere of radius $\pi$, where $C_{p} \subset M_{p}$ is by definition the tangent cut locus of $p$.

If $\gamma:[0, \pi] \rightarrow M$ is a geodesic with $\gamma(0)=p,\|\dot{\gamma}\|=1$, then $\gamma(\pi) \in C(p)$ and from $\operatorname{Ric}_{M} \geqslant 1, \gamma(\pi)$ is conjugate to $p$ along $\gamma$. It follows from the index comparison theorem (see [10,p. 178]) together with $\operatorname{Ind}_{\gamma \mid[Q \pi)} \geqslant 0$ that if $E$ is a unit parallel field along $\gamma$ with $\langle E, \dot{\gamma}\rangle=0$, then $\sin t E(t)$ is a Jacobi field along $\gamma$. Thus the multiplicity of the first conjugate point $\gamma(\pi)$ to $p$ along $\gamma$ is $m-1$. This fact implies that $C(p)=\{q\}$. Because the sectional curvature determined by the plane section 
$(\dot{\gamma}(t), E(t))$ is $1, M$ is isometric to the standard unit sphere by the composed exponential mappings.

3. Estimate of contractibility radius. Let $N$ be a complete manifold of dimension $\boldsymbol{n}$. For a fixed point $x \in N$ consider the distance function $d_{x}: N \rightarrow R, d_{x}(y)=d(x, y)$. A point $y \in N$ is by definition a noncritical point of $d_{x}$ if the set of all unit vectors tangent to minimizing geodesics from $y$ to $x$ is contained in an open half space of $N_{x}$. A point $y \in N$ is by definition a critical point of $d_{x}$ if for any nonzero tangent vector $u \in N_{x}$ there is a minimizing geodesic from $y$ to $x$ whose tangent vector at $y$ makes an angle with $u$ not greater than $\pi / 2$. Obviously a critical point $y$ of $d_{x}$ belongs to the cut locus $C(x)$ of $x$.

If $\bar{B}_{r}(x)$ contains no critical point of $d_{x}$ except at the origin $x$ of the ball, then $d_{x} \mid \bar{B}_{r}(x)$ can be approximated by smooth functions which have no critical point except at the origin $x$. In fact, let $2 r_{0}$ be the radius of a strongly convex ball around $x$. The smooth approximations of $d_{x}$ are obtained as follows: Let $\Phi: R \rightarrow R$ be a smooth function whose support is in $[-1,1]$ such that it is constant 1 around 0 and such that $\int_{v \in R^{n}} \Phi(\|v\|)=1$. For a sufficiently small positive $\rho$, define $\left(d_{x}\right)_{\rho}: N \rightarrow R$ by

$$
\left(d_{x}\right)_{\rho}(q):=\rho^{-n} \int_{v \in N_{q}} d_{x}\left(\exp _{q} v\right) \Phi(\|v\| / \rho) d v
$$

where $d v$ is the Riemannian volume on $N_{q}$. It has been proved in [8] that $\left(d_{x}\right)_{p}$ is smooth and $\left\{\left(d_{x}\right)_{\rho}\right\}_{\rho}$ converge uniformly to $d_{x}$ as $\rho \rightarrow 0$. Moreover, it has been proved in [12] that $\left(d_{x}\right)_{\rho} \mid \bar{B}_{r}(x)-\bar{B}_{r_{0}}(x)$ has nonvanishing gradient and the gradient vectors are transversal to $\partial B_{r_{0}}(x)$. And hence $\bar{B}_{r}(x)$ is contractible to $x$.

The contractibility radius $c(x)$ at $x \in N$ is defined as

$$
c(x):=\sup \left\{r ; \bar{B}_{r}(x) \text { is contractible to } x\right\} .
$$

LeMma 3.1. Let $N$ be a complete manifold. The contractibility radius function $c$ : $N \rightarrow R$ has the following properties: (1) For every $x \in N c(x)$ is not less than the positive minimum critical value $=: c_{1}(x)$ of $d_{x}$; (2) The positive minimum critical value is lower semicontinuous on $N$.

Proof. If $\bar{B}_{r}(x)$ contains no critical point of $d_{x}$, then the above argument shows that $c(x) \geqslant r$. This proves (1). Let $\left\{x_{i}\right\}$ be a sequence of points on $N$ which tends to $x \in N$, and for each $i$ let $y_{i}$ be a critical point of $d_{x_{i}}$ with $d\left(x_{i}, y_{i}\right)=c_{1}\left(x_{i}\right)$. If $\left\{y_{i}\right\}$ tends to $y \in N$, then $y$ is a critical point of $d_{x}$. Therefore $\liminf c_{1}\left(x_{i}\right) \geqslant c_{1}(x)$.

It follows from Lemma 3.1 that a lower bound of the contractibility radius on $N$ is obtained by the infimum of the positive minimum critical values of distance functions.

Now let $M$ be a complete manifold of dimension $m$ whose Ricci curvature and volume satisfy $\operatorname{Ric}_{M} \geqslant 1, v(M) \geqslant c_{m}-\eta(m, \varepsilon)$. The first observation to give an estimate of contractibility radius for a certain class of manifolds with the above properties is this: Under the assumptions for Ricci curvature and volume of $M$ as stated above, each point $x \in M$ has the property that

$$
l(x):=\max \{d(x, y) ; y \in M\} \geqslant \pi-\varepsilon .
$$


Indeed, it follows from the basic lemma together with $\bar{B}_{l(x)}(x)=M$ that $v\left(\tilde{B}_{l(x)}\right) \geqslant$ $v(M) \geqslant c_{m}-\eta(m, \varepsilon)$. Since $v\left(\tilde{B}_{l(x)}\right)=\eta(m, l(x)), c_{m}-\eta(m, \varepsilon)=\eta(m, \pi-\varepsilon)$ and since $\eta(m, \varepsilon)$ is strictly increasing with $\varepsilon, l(x) \geqslant \pi-\varepsilon$.

As a straightforward consequence of the basic lemma, we have

LEMMA 3.2. If $M$ is a complete manifold of dimension $m$ whose Ricci curvature and volume satisfy $\operatorname{Ric}_{M} \geqslant 1, v(M)>c_{m} / 2$, then $M$ is simply connected.

The next observation is to see what will happen when $c(x)$ is small roughly speaking. Since a critical point of $d_{x}$ belongs to $C(x)$, it will turn out that the appearance of such a critical point will give less contribution to the volume of concentric balls around $x$ which contain critical points of $d_{x}$. But if $v(M)$ is bounded below, then the total volume of the compact set in $M_{x}$ which is star-shaped with respect to the origin and whose boundary consists of $C_{x}$ is also bounded below. This phenomenon will be interpreted as follows.

Proposition 3.3. Let $\varepsilon \in(0, \pi)$ be a given constant. Assume that $\mathrm{Ric}_{M} \geqslant 1$ and $v(M) \geqslant c_{m}-\eta(m, \varepsilon)$. For every point $x \in M$ and a number $\theta \in(0, \pi)$ and for every $v \in S_{x}$ let $V(v ; \theta):=\left\{w \in M_{x} ; \Varangle(v, w) \leqslant \theta\right\}$. Then there exists a positive smooth function $r \rightarrow \theta(r, m, \varepsilon), 0<r<\pi-\varepsilon$, such that if every $w \in V(v ; \theta) \cap C_{x}$ has norm $\|w\| \leqslant r$, then $\theta \leqslant \theta(r, m, \varepsilon) . \theta(r, m, \varepsilon)$ is obtained as the solution of

$$
c_{m-2} \int_{0}^{\theta(r, m, \varepsilon)} \sin ^{m-2} t d t=\eta(m, \varepsilon) / \int_{r}^{\pi} \sin ^{m-1} t d t .
$$

Proof. As is seen in the first observation, $l(x) \geqslant \pi-\varepsilon$ holds for every $x \in M$. The area of $V(v ; \theta) \cap S_{x}$ is $\eta(m-1, \theta)$. Thus an upper bound for $v(M)$ is given by

$$
\eta(m-1, \theta) \int_{0}^{r} \sin ^{m-1} t d t+\eta(m-1, \pi-\theta) \int_{0}^{\pi} \sin ^{m-1} t d t \geqslant v(M) .
$$

Since $v(M) \geqslant c_{m}-\eta(m, \varepsilon)$, the above inequality reduces to

$$
\eta(m-1, \theta) \int_{r}^{\pi} \sin ^{m-1} t d t \leqslant \eta(m, \varepsilon) .
$$

The desired $\theta(r, m, \varepsilon)$ is obtained by solving

$$
c_{m-2} \int_{0}^{\theta} \sin ^{m-2} t d t \int_{r}^{\pi} \sin ^{m-1} t d t=\eta(m, \varepsilon) .
$$

The function $\theta(r, m, \varepsilon)$ has the following properties: (1) For every fixed $r \in(0, \pi$ $-\varepsilon) \lim _{\varepsilon \downarrow 0} \theta(r, m, \varepsilon)=0$ and $\varepsilon \rightarrow \theta(r, m, \varepsilon)$ is monotone increasing. (2) For every fixed $\varepsilon \in(0, \pi) 0<\lim _{r \downarrow 0} \theta(r, m, \varepsilon)<\eta^{-1}\left[\eta(m, \varepsilon) / \int_{0}^{\pi} \sin ^{m-1} t d t\right]$.

Let $\delta \in[\varepsilon, \pi / 3)$ be a fixed number and set $r_{1}=\pi-3 \delta$. It follows from Proposition 3.3 together with the continuity of the map $u \in S_{x} \rightarrow$ the distance from $x$ to the cut point of $x$ along the goedesic $t \rightarrow \exp _{x} t u$ that for every $x \in M$ and for every $u \in S_{x}$ there exists a $w \in S_{x}$ with the properties that $\Varangle(u, w) \leqslant \theta\left(r_{1}, m, \varepsilon\right)$ and that the cut point $\exp _{x} t_{1} w$ to $x$ along the geodisic $t \rightarrow \exp _{x} t w$ appears at $t_{1} \geqslant r_{1}$. Then the Toponogov comparison theorem will be applied to obtain a lower bound for $c_{1}$ : $M \rightarrow R$. Thus an additional assumption for the sectional curvature will be needed. 
THEOREM 3.4. Let $m$ be a positive integer and let $\kappa>0$ and $\varepsilon \in(0, \pi / 3)$ be given constants. Then there exists for a fixed number $\delta \in[\varepsilon, \pi / 3)$ a constant $c_{\delta}(m, \kappa, \varepsilon)>0$ such that if $M$ is a complete manifold of dimension $m$ whose curvatures and volume satisfy

$$
\operatorname{Ric}_{M} \geqslant 1, \quad K_{M} \geqslant-\kappa^{2}, \quad v(M) \geqslant \eta(m, \pi-\varepsilon),
$$

then $c_{1}(x) \geqslant c_{\delta}(m, \kappa, \varepsilon)$ for every point $x \in M$. The constant is given by

$$
c_{\delta}(m, \kappa, \varepsilon)=\min \left[\pi-3 \delta, \kappa^{-1} \tanh ^{-1}\{\tanh (\pi-3 \delta) \kappa \cdot \cos \theta(\pi-3 \delta, m, \varepsilon)\}\right] .
$$

Proof. Let $x \in M$ be a fixed point and let $y \in M$ be a critical point of $d_{x}$ with the positive minimum critical value $r_{0}=c_{1}(x)$. Let $u \in S_{x}$ be the unit vector tangent to a minimizing geodesic $\gamma_{u}:\left[0, r_{0}\right] \rightarrow M$ with $\gamma_{u}(0)=x, \gamma_{u}\left(r_{0}\right)=y$. The above argument shows that there is a $w \in S_{x}$ with the properties: $\Varangle(u, w) \leqslant \theta\left(r_{1}, m, \varepsilon\right)$ and $\gamma_{w}$ has the cut point to $x$ along it at $\gamma_{w}\left(t_{1}\right)$ with $t_{1} \geqslant r_{1}$. If $r_{0} \geqslant r_{1}$ then nothing is left to prove.

Assume that $r_{0}<r_{1}$. The Toponogov comparison theorem implies that if $\alpha=$ $\Varangle(u, w)$ and if $r_{2}=d(y, z)$, where $z=\gamma_{w}\left(t_{1}\right)$, then

$$
\cosh r_{2} \kappa \leqslant \cosh t_{1} \kappa \cdot \cosh r_{0} \kappa-\sinh t_{1} \kappa \cdot \sinh r_{0} \kappa \cdot \cos \alpha .
$$

Since $y$ is a critical point of $d_{x}$, there exists for a minimizing geodesic from $y$ to $z$, a minimizing geodesic from $y$ to $x$ (possibly different from $\gamma_{u}$ ) whose angle at $y$ is not greater than $\pi / 2$. Thus the Toponogov theorem again implies for this triangle to get

$$
\cosh t_{1} \kappa \leqslant \cosh r_{0} \kappa \cdot \cosh r_{2} \kappa .
$$

Eliminate $r_{2}$ from the above inequalities to obtain

$$
\cosh t_{1} \kappa \cdot \tanh r_{0} \kappa \geqslant \cos \alpha .
$$

Insert $\alpha \leqslant \theta\left(r_{1}, m, \varepsilon\right)$ and $t_{1} \geqslant r_{1}=\pi-3 \delta$ to complete the proof.

It should be noted that for every fixed $\delta \in[\varepsilon, \pi / 3), \lim _{\varepsilon \downarrow 0} c_{\delta}(m, \kappa, \varepsilon)=\pi-3 \delta$.

4. The proof of Main Theorem and remarks. By means of the generalized Schoenflies theorem, it suffices for the proof of our main theorem to exhibit $M$ as a union of two open disks. In fact the open disks are obtained as contractible metric balls.

Proof of Main Theorem. It follows from Theorem 3.4 that if $r \in(0, \pi)$ is arbitrarily given, then there exists an $\varepsilon \in(0, \pi / 3)$ and a $\delta \in[\varepsilon, \pi / 3)$ such that $r \leqslant c_{\delta}(m, \kappa, \varepsilon)$.

Let $p, q \in M$ be a pair of points such that $d:=d(p, q)=d(M)$. Then $\pi-\varepsilon \leqslant d$ $\leqslant \pi$. The minimal radius $R$ of closed balls around $p$ and $q$ by which $M$ is covered satisfies $d / 2 \leqslant R \leqslant d$ and $R=\max \{d(p, y) ; y \in M, d(p, y)=d(q, y)\}$. It follows from the basic lemma that if $x$ is a point with $d(p, x)=d(q, x)=R$, then

$$
\begin{aligned}
v(M) & \geqslant v\left(B_{d / 2}(p)\right)+v\left(B_{d / 2}(q)\right)+v\left(B_{R-d / 2}(x)\right) \\
& \geqslant\left(v(M) / c_{m}\right) \times\left[2 v\left(\tilde{B}_{d / 2}\right)+v\left(\tilde{B}_{R-d / 2}\right)\right] .
\end{aligned}
$$

Thus

$$
c_{m} \geqslant c_{m-1}\left[2 \int_{0}^{(\pi-\epsilon) / 2} \sin ^{m-1} t d t+\int_{0}^{R-\pi / 2} \sin ^{m-1} t d t\right] .
$$


Let $R_{0}=R(m, \varepsilon)$ be the solution of

$$
\int_{(\pi-\varepsilon) / 2}^{(\pi+\varepsilon) / 2} \sin ^{m-1} t d t=\int_{0}^{R_{0}-\pi / 2} \sin ^{m-1} t d t
$$

Then $R_{0} \geqslant R$ and $\lim _{\varepsilon \downarrow 0} R(m, \varepsilon)=\pi / 2$ and $\varepsilon \rightarrow R(m, \varepsilon)$ is strictly monotone increasing. The desired $\varepsilon=\varepsilon(m, \kappa)$ is obtained as follows. Let $\delta \in(0, \pi / 6)$ be a fixed number and let $\varepsilon \in(0, \delta]$. Then $\lim _{\varepsilon \downarrow 0} c_{\delta}(m, \kappa, \varepsilon)=\pi-3 \delta>\pi / 2=$ $\lim _{\varepsilon \downarrow 0} R(m, \varepsilon)$. Because $c_{\delta}(m, \kappa, \varepsilon)$ is monotone decreasing with $\varepsilon \in(0, \delta]$ and because $R(m, \varepsilon)$ is monotone increasing with $\varepsilon$, there exists a unique $\varepsilon(m, \kappa)$ such that if $\varepsilon \in(0, \varepsilon(m, \kappa))$ then $R(m, \varepsilon)<c_{\delta}(m, \kappa, \varepsilon)$. If $r:=\left(R(m, \varepsilon)+c_{\delta}(m, \kappa, \varepsilon)\right) / 2$, then $r<c_{\delta}(m, \kappa, \varepsilon)$ implies that $B_{r}(p)$ and $B_{r}(q)$ are open disks and $r>R(m, \varepsilon)$ implies that they cover $M$.

REMARK 1. If we apply Gromov's technique developed in [11] to our case, then the sum of Betti numbers is bounded as follows. For given constants $m, \kappa>0$ and $\varepsilon \in(0, \pi / 3)$ consider the class of all complete manifolds of dimension $m$ satisfying $\operatorname{Ric}_{M} \geqslant 1, K_{M} \geqslant-\kappa^{2}, v(M) \geqslant c_{m}-\eta(m, \varepsilon)$. Let $2 c:=c_{\varepsilon}(m, \kappa, \varepsilon)$. Then each $M$ in the class has the property that every point $x$ on $M$ has a contractible metric ball of radius at least $2 c$. The minimal covering argument (see [4,11 and 19]) implies that $M$ is covered by at most $N(m, \kappa, \varepsilon):=\left[c_{m} / v\left(B_{c}\right)\right]+1$ contractible balls. Since every contractible ball has content $1, \operatorname{cont}(M)=\Sigma^{m} b_{i}(M ; F) \leqslant(m+1) 2^{N(m, \kappa, \varepsilon)}$ follows from the topological lemma in [11].

REMARK 2. In the proof of finiteness theorems due to Weinstein [19] and Cheeger [4], it is essential to find a uniform positive lower bound for convexity radius of a certain class of complete manifolds. The assumption for an upper bound of sectional curvature of the class plays an important role to obtain such a uniform positive lower bound for convexity radius. However in our case it is not easy to find such a lower bound because there is no assumption for the upper bound of sectional curvature. It is difficult to see when a contractible metric ball becomes a convex ball. Also it is hard to control the intersections of contractible metric balls. And this point makes it difficult to prove finiteness of homotopy types of the class.

Let us assume that two manifolds $M$ and $M^{\prime}$ of the same dimension have the same number of topological disks $B_{1}, \ldots, B_{N} \subset M$ and $B_{1}^{\prime}, \ldots, B_{N}^{\prime} \subset M^{\prime}$ such that they satisfy: (1) $\cup B_{i}=M$ and $\cup B_{i}^{\prime} M^{\prime}$, (2) for every $1 \leqslant i_{1}, \ldots, i_{k} \leqslant N$ the number of components of the intersection $B_{i_{1}} \cap B_{i_{2}} \cap \cdots \cap B_{i_{k}}$ is equal to that of the intersection $B_{i_{1}}^{\prime} \cap B_{i_{2}}^{\prime} \cap \ldots \cap B_{i_{k}}^{\prime}$. The question is if $M$ and $M^{\prime}$ have the same homotopy type. There is a counterexample of 3-dimensional manifolds $M$ and $M^{\prime}$ with the covers by the same number of disks which satisfy the above properties and the homotopy type of $M$ is different from that of $M^{\prime}$. Such an example was first discovered by Tsukui for the lens space $L^{3}(3 ; 1)$ of type $(3 ; 1)$ and a connected sum $P^{3} \# P^{3}$ of real projective spaces. Then a simpler example has been furnished by T. Kaneto for $S^{3}$ and $P^{3}$ as follows.

EXAmple. Let $T_{1}$ and $T_{2}$ be 3-dimensional solid tori. It follows from the Hopf fibration that $S^{3}$ is obtained by attaching $\partial T_{1}$ and $\partial T_{2}$ with an attaching map $\phi$ : $\partial T_{1} \rightarrow \partial T_{2}$ as $S^{3} \approx T_{1} \cup_{\phi} T_{2} . T_{1}$ and $T_{2}$ are decomposed into $\hat{B}_{1}, \hat{B}_{2}$ and $\hat{B}_{3}, \hat{B}_{4}$ such that $\hat{B}_{1} \cap \hat{B}_{2}$ and $\hat{B}_{3} \cap \hat{B}_{4}$ consist of two closed 2-disks, as indicated in the figure. 

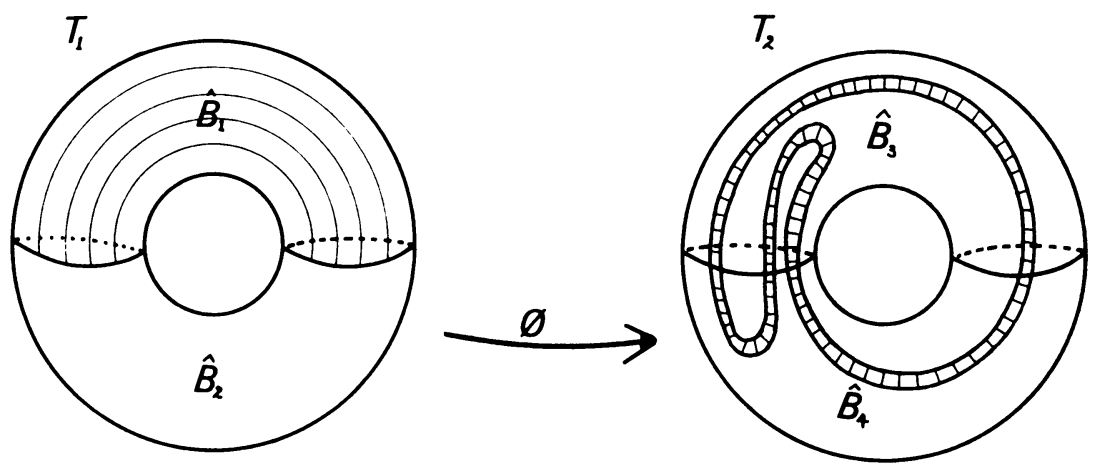

For each $i=1, \ldots, 4$ let $B_{i}$ be a neighborhood of $B_{i}$ in $S^{3}$ which is homeomorphic to an open 3-disk. Choose an attaching map $\phi$ in such a way that $\phi$ maps $\partial B_{1}$ into $\partial T_{2}$ whose image is indicated by the shaded region and $\partial B_{2}$ onto the unshaded region of $\partial T_{2}$. Then $\left\{B_{1}, B_{2}, B_{3}, B_{4}\right\}$ forms a covering of $S^{3}$ by disks and satisfies: (1) for every $i, j=1, \ldots, 4$ with $i \neq j, B_{i} \cap B_{j}$ consists of two 3-cells, (2) for every $i, j, k=$ $1, \ldots, 4$ with $i \neq j \neq k \neq i, B_{i} \cap B_{j} \cap B_{k}$ consists of four 3-cells, (3) $B_{1} \cap B_{2} \cap B_{3}$ $\cap B_{4}$ consists of eight 3-cells.

Let $B \subset R^{3}$ be the unit ball around the origin. $P^{3}$ is obtained by identifying the boundary $\partial B=S^{2}$ via the antipodal map. For a fixed number $r \in(0,1)$ and for a fixed straight line $L$ passing through the origin, let $U$ be an $r$-tubular neighborhood of $L$ in $R^{3} . P^{3}$ is then decomposed as a union of two solid tori $T_{1}^{\prime}$ and $T_{2}^{\prime}$ which are obtained by identifying $\partial B \cap U$ and $\partial B-U$ by the antipodal map. Obviously $\partial U \cap B$ is homeomorphic to $T^{2}$ after the identification. Every circle on $\partial T_{2}^{\prime}$ which is obtained by a line segment in $\partial U \cap B$ parallel to $L$ turns around $\partial T_{2}^{\prime}$ twice.
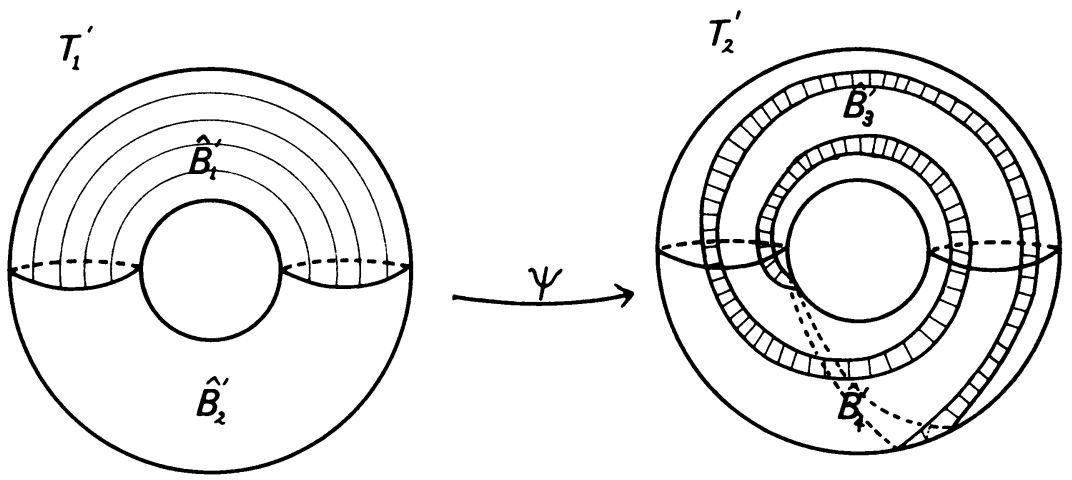

$T_{1}^{\prime}$ and $T_{2}^{\prime}$ are decomposed into $\hat{B}_{1}^{\prime}, \hat{B}_{2}^{\prime}$ and $\hat{B}_{3}^{\prime}, \hat{B}_{4}^{\prime}$ respectively such that $\hat{B}_{1}^{\prime} \cap \hat{B}_{2}^{\prime}$ and $\hat{B}_{3}^{\prime} \cap \hat{B}_{4}^{\prime}$ consist of two closed 2-disks, as indicated in the figure. For each $i=1, \ldots, 4$ let $B_{i}^{\prime}$ be a neighborhood of $\hat{B}_{i}^{\prime}$ in $P^{3}$ which is homeomorphic to an open 3-disk. Choose an attaching map $\psi: \partial T_{1}^{\prime} \rightarrow \partial T_{2}^{\prime}$ which sends $\partial B_{1}^{\prime}$ onto the shaded region of $\partial T_{2}^{\prime}$ and $\partial B_{2}^{\prime}$ onto the unshaded region of $\partial T_{2}^{\prime}$. Then $\left\{B_{1}^{\prime}, B_{2}^{\prime}, B_{3}^{\prime}, B_{4}^{\prime}\right\}$ is a covering of $P^{3}$ by disks and satisfies: (1) for every $i, j=1, \ldots, 4$ with $i \neq j, B_{i}^{\prime} \cap B_{j}^{\prime}$ consists of two 3-cells, (2) for every $i, j, k=1, \ldots, 4$ with $i \neq j \neq k \neq i, B_{i}^{\prime} \cap B_{j}^{\prime} \cap$ $B_{k}^{\prime}$ consists of four 3-cells, (3) $B_{1}^{\prime} \cap B_{2}^{\prime} \cap B_{3}^{\prime} \cap B_{4}^{\prime}$ consists of eight 3-cells. 
Thus $S^{3}$ and $P^{3}$ have the same type of covering by disks, however they do not have the same homotopy type.

\section{REFERENCES}

1. M. Berger, Les variétés riemanniennes (1/4)-pincées, Ann. Scuola Norm. Sup. Pisa Cl. Sci. (4) 14 (1960), 161-170.

2. R. Bishop and R. L. Crittenden, Geometry of manifolds, Academic Press, New York, 1964.

3. M. Brown, A proof of the generalized Schoenflies theorem, Bull. Amer. Math. Soc. 66 (1960), 74-76.

4. J. Cheeger, Finiteness theorems for riemannian manifolds, Amer. J. Math. 92 (1970), 61-74.

5. J. Cheeger and D. Ebin, Comparison theorems in Riemannian geometry, North-Holland, Amsterdam and New York, 1975.

6. J. Cheeger and D. Gromoll, The splitting theorem for manifolds of nonnegative Ricci curvature, J. Differential Geom. 6 (1971), 119-129.

7. S. Y. Cheng, Eigenvalue comparison theorem and its geometric applications, Math. Z. 143 (1975), 289-297.

8. R. Greene and $\mathrm{H}$. Wu, On the subharmonicity and plurisubharmonicity of geodesically convex functions, Indiana Univ. Math. J. 22 (1973), 641-653.

9.

10. D. Gromoll, W. Klingenberg and W. Meyer, Riemannsche Geometrie im Grossen, Springer-Verlag, Berlin, Heidelberg and New York, 1966.

11. M. Gromov, Curvature, diameter and Betti numbers, Comment. Math. Helv. 56 (1981), 179-195.

12. K. Grove and K. Shiohama, A generalized sphere theorem, Ann. of Math. (2) 106 (1977), 201-211.

13. W. Klingenberg, Über Riemannsche Mannigfaltigkeiten mit positiver Krümmung, Comment. Math. Helv. 35 (1961), 47-54.

14. S. B. Myers, Riemannian manifolds with positive mean curvature, Duke Math. J. 8 (1941), 401-404.

15. H. Rauch, A contribution to differential geometry in the large, Ann. of Math. (2) 54 (1951), 38-55.

16. T. Rushing, Topological embeddings, Academic Press, New York and London, 1973.

17. B. Schoen and S. T. Yau, Complete three dimensional manifolds with positive Ricci curvature and scalar curvature, Seminar on Differential Geometry, Ann. Math. Studies, no. 102, Univ. of Princeton. Princeton, N.J., 1982, pp. 209-228.

18. V. A. Toponogov, Riemannian spaces having their curvature bounded below by a positive number, Amer. Math. Soc. Transl. (2) 37 (1964), 291-336; translated from Uspehi Mat. Nauk 14 (1959).

19. A. Weinstein, On the homotopy of positively pinched manifolds, Arch. Math. (Basel) 18 (1967), $523-524$.

Department of Mathematics, University of TSUkUba, Sakura-mura, Ibaraki, 305-Japan 